\title{
Increased Neurogenesis in a Model of Electroconvulsive Therapy
}

\author{
Torsten M. Madsen, Alexandra Treschow, Johan Bengzon, Tom G. Bolwig, \\ Olle Lindvall, and Anders Tingström
}

Background: Electroconvulsive therapy (ECT) is a widely used and efficient treatment modality in psychiatry, although the basis for its therapeutic effect is still unknown. Past research has shown seizure activity to be a regulator of neurogenesis in the adult brain. This study examines the effect of a single and multiple electroconvulsive seizures on neurogenesis in the rat dentate gyrus.

Methods: Rats were given either a single or a series of 10 electroconvulsive seizures. At different times after the seizures, a marker of proliferating cells, Bromodeoxyuridine $(B r d U)$, was administered to the animals. Subsequently, newborn cells positive for BrdU were counted in the dentate gyrus. Double staining with a neuron-specific marker indicated that the newborn cells displayed a neuronal phenotype.

Results: A single electroconvulsive seizure significantly increased the number of new born cells in the dentate gyrus. These cells survived for at least 3 months. A series of seizures further increased neurogenesis, indicating a dose-dependent mechanism.

Conclusions: We propose that generation of new neurons in the hippocampus may be an important neurobiologic element underlying the clinical effects of electroconvulsive seizures. Biol Psychiatry 2000;47:1043-1049 (C) 2000 Society of Biological Psychiatry

Key Words: Neurogenesis, seizure, BrdU, depressive disorder, hippocampus, ECT

\section{Introduction}

$\mathrm{I}^{\mathrm{n}}$ nduction of seizures in the form of electroconvulsive therapy (ECT) has been used in the treatment of psychiatric disorders for more than 60 years. Particularly in the treatment of severe major depression, evidence for

From the Neuropsychiatry Laboratory, Department of Psychiatry, H:S Rigshospitalet, Copenhagen, Denmark (TMM, TGB) and the Molecular Psychiatry Unit (ATr, ATi) and the Section of Restorative Neurology (JB, OL), Wallenberg Neuroscience Center, University Hospital, Lund, Sweden.

Address reprint requests to Tom G. Bolwig, M.D., D.Sc., Copenhagen University Hospital, Neuropsychiatric Laboratory, Department of Psychiatry, H:S Rigshospitalet-6234, DK-2100, Copenhagen, Denmark.

Received September 15, 1999; revised December 15, 1999; accepted December 17 1999. the effectiveness and superiority of ECT over other treatments is clear and convincing (Janicak et al 1985). Nonetheless, despite much effort to clarify the basis for the beneficial effects of this treatment modality, its mechanism of action is still largely unknown. The generalization of seizures that is a prerequisite for the therapeutic effect (Sackeim et al 1996) probably exerts a strong influence on diencephalic-limbic structures, the biochemistry of which is disturbed in major depression (Goodwin 1990).

Among particular structures calling for attention in relation to depression is the hippocampus. Because animal studies have demonstrated a profound effect on a number of signaling molecules, including monoamines, neuropeptides, and growth factors, in the hippocampus (Mathé 1999; Mongeau et al 1997; Nibuya et al 1995) and this region also is vulnerable to stressful stimuli and increased glucocorticoid levels (McEwen 1999), we found it relevant to focus particularly on the hippocampal formation in a study on the effects of ECT in an animal model. The hippocampus is crucial for the formation of memory (Squire 1986a), and many elderly patients display gross depression-related memory impairment (depressive pseudodementia) that disappears following recovery. Further, a decreased hippocampal volume has been reported in patients with repeated episodes of major depression (Shah et al 1998; Sheline et al 1996, 1999).

Clinical efficacy of ECT requires seizures of a longer duration than 20-30 sec for each of typically 6-10 seizures to ensure maximal response (American Psychiatric Association 1990). This treatment gives rise to transient cognitive impairment (Squire 1986b), but no other side effects have been consistently documented, although both ECT and electroconvulsive seizure (ECS), the animal model for this treatment modality, have been carefully examined for accompanying tissue damage (Devanand et al 1994; Vaidya et al 1999).

The dentate gyrus of the hippocampal formation is a site for continuos neurogenesis during adult life in animals, including humans (Altman and Das 1965; Eriksson et al 1998; Gould et al 1998). Proliferation, differentiation, and survival of dentate granule neurons are influenced by a number of physiologic and environmental conditions 
(Kempermann et al 1997b; van Praag et al 1999). In rodents, brain insults such as global ischaemia (Liu et al 1998) and experimentally induced seizures markedly increase hippocampal neurogenesis, although primarily following extended periods of seizure or in association with neuronal damage (Bengzon et al 1997; Parent et al 1998).

In our study, we have analyzed neurogenesis in the dentate gyrus of rats following ECS. The objectives were first to explore the possibility that a single ECS triggers neurogenesis and, if so, whether repeated ECS would further increase cell proliferation. Second, we hoped to determine whether the newly formed neurons were short lived or exhibited long-term survival. Finally, we hoped to determine if ECS leads to neuronal death by an apoptotic mechanism as previously reported for kindling-evoked seizures (Bengzon et al 1997). To assess cell proliferation, we used the thymidine analogue, bromodeoxyuridine (BrdU), which is incorporated into DNA in dividing cells in the S-phase and can be detected by immunohistochemistry. Neurons were identified by the presence of the neuron-specific marker NeuN. Apoptosis was detected by enzymatic labeling of cleaved DNA fragments in the apoptotic cells.

\section{Methods and Materials}

\section{Animals and Design of Study}

We used adult male Wistar rats (Møllegaard Breeding Center, Ll. Skensved, Denmark), which weighed between 250 and $400 \mathrm{~g}$ at the beginning of the study. The animals were housed three per cage and were allowed free access to ordinary lab chow and water.

The time course of maximal proliferation was assessed by two injections of BrdU with a 12-hour intervals, starting at different time points $(0,3,5,7$, and 9 days) after $\operatorname{ECS}(n=5$ per time point). Rats survived 48 hours after the last injection of BrdU. As maximal cell proliferation seemed to occur on days 3 and 5 after the ECS, BrdU was administered from day 3 to day 6 in the single ECS experiment. Each rat received six injections of BrdU at 12-hour intervals, starting 72 hours after the administration of a single ECS or sham treatment. The animals were then allowed to survive for 1 or 3 months ( $n=5$ in each group). For the repeated ECS experiment, four groups ( $n=7$ in each) were used. Starting on day 1 , the animals received either one ECS, 10 ECSs (one daily for 10 days), five ECSs (on days 1, 3, 5, 7, and 9), or 10 sham treatments (one daily for 10 days). BrdU was administered twice daily from day 1 to day 21 of the experiment (a total of 42 injections). The animals were allowed to survive for 14 days after the last injection of BrdU.

In a separate experiment to detect apoptotic cells, rats were administered either one or a series of 10 ECSs (one daily) or given corresponding sham treatments. Animals were analyzed 2 and 4 hours after the last ECS ( $n=5$ in each of the groups).

\section{Administration of Electroconvulsive Seizures}

Electroconvulsive seizures were delivered through earbar electrodes $(50 \mathrm{~mA}, 0.5 \mathrm{sec}, 50 \mathrm{~Hz}$ unidirectional square wave pulses) and consistently resulted in a period of tonic-clonic seizure activity of less than $1 \mathrm{~min}$. Sham-treated rats were handled identically to the ECS treated animals except that no current was passed.

\section{Administration of Bromodeoxyuridine}

Bromodeoxyuridine was dissolved in potassium phosphate buffered saline (KPBS) in all experiments and was administered intraperitoneally in a dose of $37.5 \mathrm{mg} / \mathrm{kg}$ per injection.

\section{Detection of Neurogenesis}

Animals were anaesthetized with Equithesin (SAD, Copenhagen, $3.3 \mathrm{~mL} / \mathrm{kg}$ intraperitoneally) and transcardially perfused with heparinized saline for $2 \mathrm{~min}$, followed by $4 \%$ paraformaldehyde for $13 \mathrm{~min}$. The brains were removed from the skull and allowed to postfix in the fixative overnight. Before sectioning on a freezing microtome, the brains were transferred to $20 \%$ sucrose in phosphate buffered saline (KPBS) until they sunk. The brains were cut in the frontal plane through the hippocampus in 40 - $\mu$-thick sections.

Sections were washed three times in KPBS and then exposed to $1 \mathrm{M} \mathrm{HCl}$ at $65^{\circ} \mathrm{C}$ for $30 \mathrm{~min}$. After three washes in KPBS $0.25 \%$ Triton $\mathrm{X}-100$, the sections were incubated in blocking buffer (KPBS $+0.25 \%$ Triton X-100 [KPBS-T] $+5 \%$ normal donkey serum (Harlan Sera-lab, Belton, UK) $+5 \%$ normal horse serum [Sigma, St. Louis]) for 1 hour at room temperature. The sections were then exposed to the primary antibody solution (blocking buffer $+1 \%$ rat-anti-BrdU (Harlan Sera-lab, MAS $250 p)+1 \%$ mouse anti-NeuN (MAB377, Chemicon, Temecula, CA) for 36 hours at $4{ }^{\circ} \mathrm{C}$ and slow shaking. Then, after two washes with KPBS-T, sections were again blocked with two washes with KPBS-T $+2 \%$ normal donkey serum (Harlan Sera-lab) $+2 \%$ normal horse serum. Then sections were incubated in the same blocking buffer with the secondary antibodies $(0.5 \%$ Cy-3 donkey-anti rat IgG [Jackson 712-165153, Jackson ImmunoResearch, West Grove, PA] and $0.5 \%$ biotin horse-anti-mouse IgG [Vector BA-2001, Vector Laboratories, Burlingame, CA]) for 2 hours in the dark. Finally, before three washes in KPBS, the sections were incubated with KPBS-T and $0.4 \%$ of flourescine avidin D (Vector A 2001) for 2 hours. After drying and mounting in polyvinyl alcohol-1-4-diazabicyclo-[2,2,2]-octane mounting medium, sections were analyzed by fluorescence or confocal microscopy.

\section{Detection of Apoptosis}

Rats were rapidly decapitated, the brains removed and frozen in powdered dry ice. The brains were kept at $-80^{\circ}$ until sectioning on a cryostat. To visualize apoptotic cells, the Oncor Apoptag kit (Oncor cat \#S7110) was used according to the manufacturers specifications. 

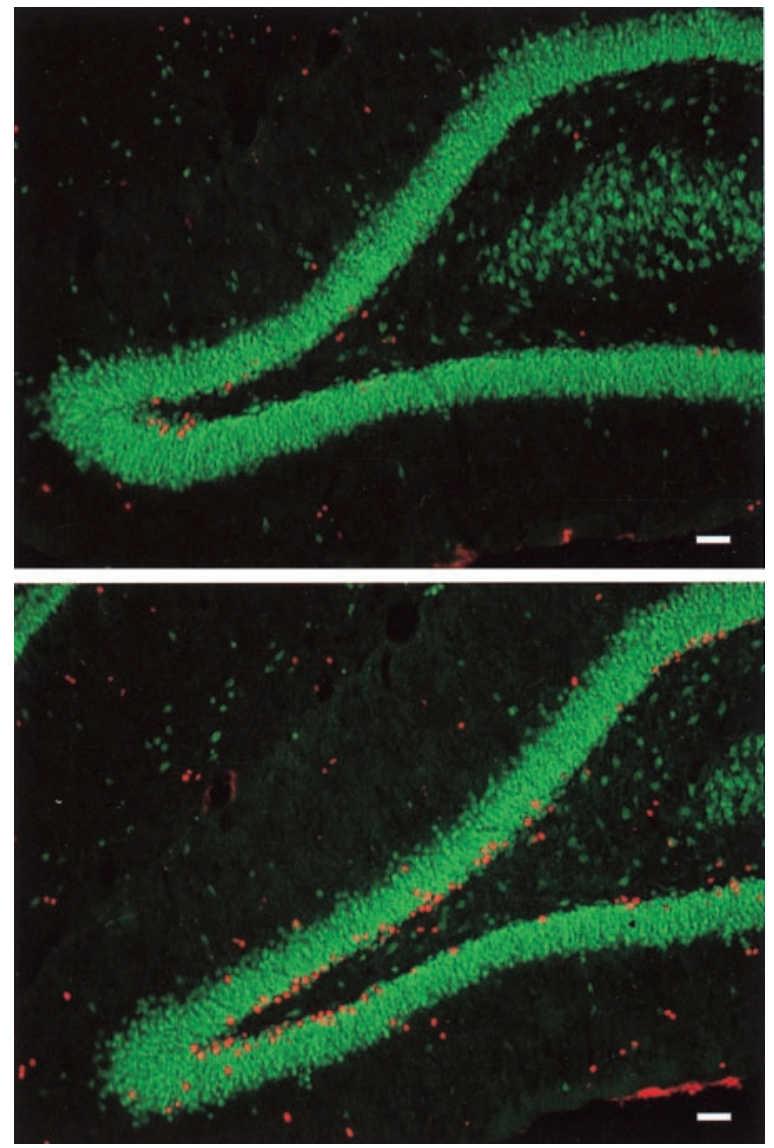

Figure 1. A single electroconvulsive seizure (ECS) stimulates cell proliferation. Double immunofluoroscence images showing a rat dentate gyrus from (top) a sham-treated animal and (bottom) an ECS treated-animal. Bromodeoxyuridine (BrdU) was injected six times with 12-hour intervals, starting 72 hours after ECS. Animals survived 1 month after the ECS treatment. Red cells are positive for the mitotic marker BrdU, and green cells are positive for the neuronal marker NeuN. Scale bar, 50 $\mu \mathrm{m}$.
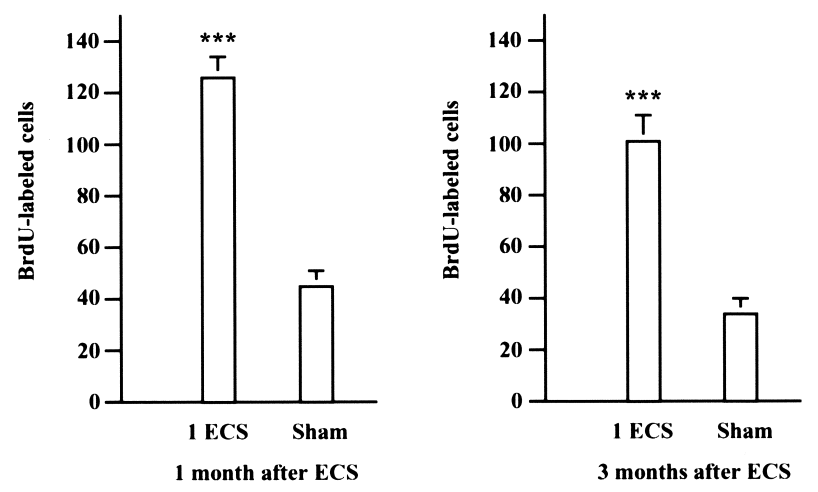

Figure 2. Survival of newborn cells. Quantification of the number of newly formed cells per $40-\mu \mathrm{m}$ section of rat dentate gyrus 1 month (left) or 3 months (right) after treatment with a single electroconvulsive seizure (ECS). Cells in the granular and hilar regions were counted. A single ECS significantly increased the number of bromodeoxyuridine-labeled cells compared with sham treatment $(* * *$ Significance, $p<.0001$; Bonferroni/Dunn correction. Error bars, SEM). No significant difference in the number of bromodeoxyuridine (BrdU)-labeled cells was observed between the 1-month survival and the 3-month survival animals.
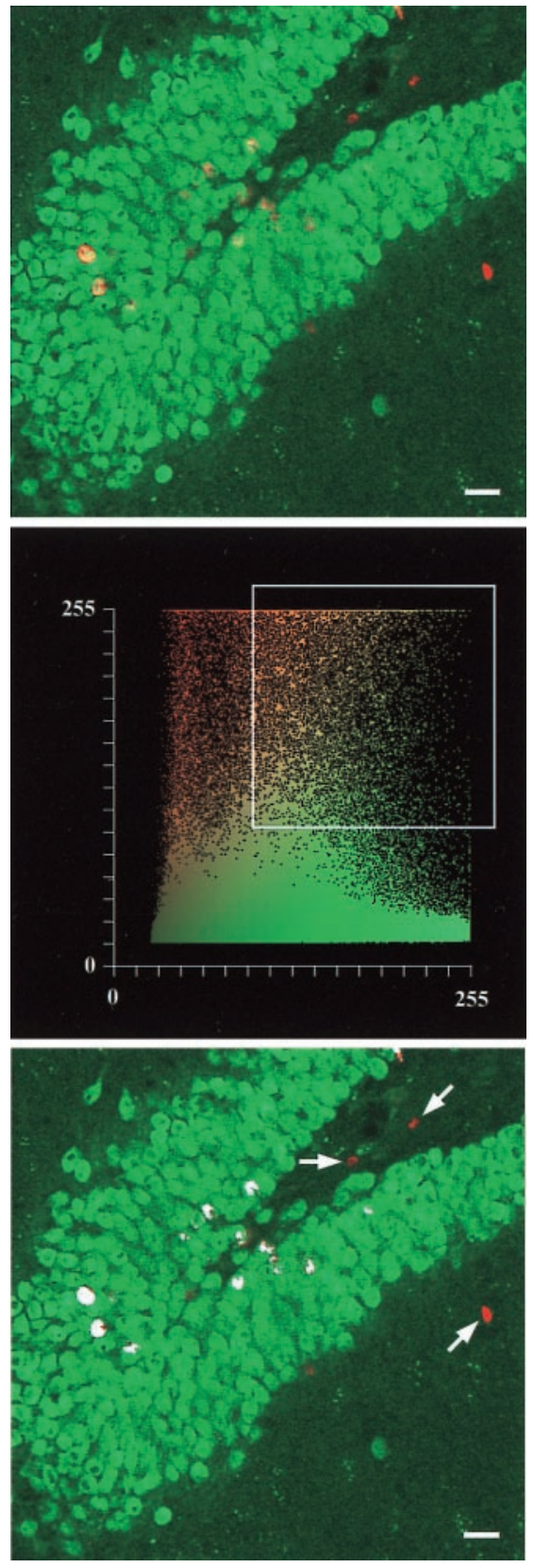

Figure 3. Newborn cells are mainly neurons. Confocal image documenting the neuronal phenotype of proliferating cells in the dentate gyrus. In the medial portion of the dentate gyrus from a rat 3 months after a single electroconvulsive seizure (top), red (bromodeoxyuridine [BrdU] positive) cells are clearly visible. To assess colocalization of BrdU and the neuronal marker NeuN (green), a cutoff level for both colors (white box, center) was chosen. Bottom: Cells with intensities above the cutoff level in both channels appear white. This documents the neuronal phenotype of the newborn cells. For comparison, arrows in the bottom panel point to BrdU-labeled cells that are not positive for NeuN. Scale bar, $20 \mu \mathrm{m}$. 


\section{Quantification of Cell Proliferation}

Sections through the dorsal hippocampus $(-3.3 \mathrm{~mm}$ to -4.5 $\mathrm{mm}$, relative to bregma; Paxinos and Watson 1986) were analyzed by observers blind to the treatment. For the single ECS experiments, all BrdU-labeled cells in the dentate gyrus including the hilus were counted. In the multiple seizure experiment, only labeled cells in the subgranular zone (SGZ) were counted. Cells lying within two cell diameters of the granule cell and hilar border were included in the SGZ count. Counting was performed using a fluorescent microscope with 40x objective. Four to six sections per animal were counted, and the counts were averaged and expressed as means per dentate gyrus or SGZ, respectively.

Confocal microscopy was performed on double-labeled sections. Z-series of sections fron the medial tip of the dentate gyrus were collected. The digitally acquired sections were of a thickness of approximately $1 \mu \mathrm{m}$, and about 20 sections were obtained by the digital sectioning. Subsequently, BrdU positive cells were analysed for the colocalisaation of NeuN immunoreactivity.

\section{Results}

\section{Proliferation and Survival of Newly Generated Cells after a Single ECS}

Cell proliferation was analyzed in the hippocampal formation. In unstimulated animals, BrdU-labeled cells were seen along the inner border of the dentate granular cell layer indicating an ongoing, basal-cell proliferation, in agreement with previous findings by other researchers (Gould et al 1999; Kempermann et al 1997a).

A single ECS increased the rate of cell proliferation in the dentate gyrus. The period of maximal cell proliferation was determined by administering BrdU at different time points after a single ECS. In the first 12 hours after an ECS, the rate of proliferation was still at baseline levels. By day 3 after the ECS, the number of BrdU-labeled cells had more than doubled, stayed elevated at 5 days but returned to control levels by day 7 (data not shown).

To examine the long-term survival of the newly formed cells, BrdU was administered within the period of maximum proliferation. One month after the administration of a single ECS, a threefold increase in the number of BrdU-labeled cells was observed (Figure 1). These cells were predominantly located in the subgranular zone on the hilar border of the dentate gyrus and often with a higher density toward the medial tip of the dentate gyrus. A survival period of 3 months yielded similar results (Figure 2), and the number of BrdU-labeled cells was not different from that in the 1-month group, indicating that the newborn cells survive for at least 3 months.

\section{Detection of Apoptosis after ECS}

As previous reports on seizures and cell proliferation have described accompanying neuronal apoptosis (Bengzon et al 1997), we examined sections from brains treated with either a single or repeated ECS for the presence of apoptotic cells. On the basis on previous kindling studies (Bengzon et al 1997), we analyzed dentate gyri from rats 2 and 4 hours after a single or the last of 10 ECSs and sham-treated control rats. In the control group, an average of 0.514 ( \pm 0.057; SEM) apoptotic nuclei were found in each $12-\mu \mathrm{m}$ section of the dentate gyrus; and 4 hours after a single ECS an average of $0.69( \pm 0.16$; SEM) was found. These figures did not differ significantly between any of the groups examined, and thus no increase in apoptotic cells was observed after the ECS treatment.

\section{Neuronal Phenotype of Newly Generated Cells after ECS}

To assess the phenotype of the newborn cells, sections from the 3-month survival group were analyzed by confocal microscopy. More than $90 \%$ of the examined population of newly formed cells in the dentate gyrus were immunopositive for both the BrdU antibody and the neuronal marker NeuN (Wolf et al 1996; Figure 3), indicating a neuronal phenotype. BrdU/NeuN doublelabeled cells were located at the hilar border of the granule cell layer, whereas the few BrdU-positive/NeuN negative cells were located in the molecular layer of the dentate gyrus and in the hippocampus proper.

\section{Cell Proliferation after Repeated ECS}

The effect of repeated ECSs was examined in four groups of animals that received ECS either once, five times (every second day for 10 days), or 10 times (once daily). Control rats were given 10 sham treatments. To cover the period of cell proliferation, an injection regimen was chosen that started the day of the first ECS and continued with BrdU injections 12 hours apart for a period of 21 days. Animals subjected to 10 ECSs had significantly more labeled cells in the granular cell layer than did control animals. These labeled cells spanned the entire extent of the subgranular zone of the dentate gyrus (Figure 4). The magnitude of proliferation in the five ECS group was between those in the single and the 10 ECS groups. In contrast to the findings described above, we observed no significant difference between the single ECS and the sham groups in this experiment. This was probably attributable to the extended period of BrdU labeling compared with the relatively short interval of enhanced cell proliferation seen following a single ECS.

\section{Discussion}

Our study demonstrates a significant increase in the number of newly generated hippocampal neurons in an 

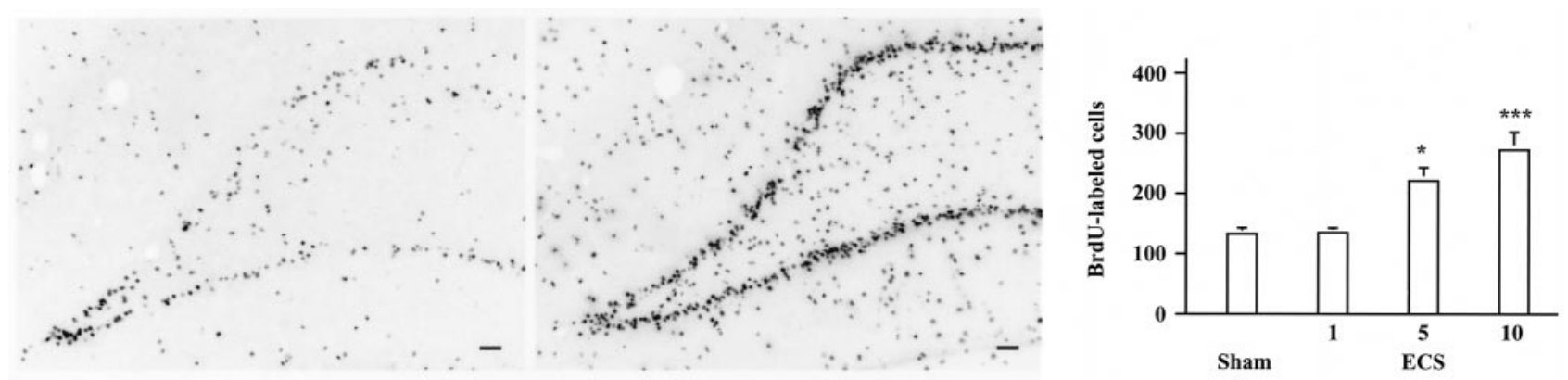

Figure 4. Increased cell proliferation with increased number of electroconvulsive seizures (ECSs). Rat dentate gyrus after 10 sham stimulations (left) and after 10 ECSs (center). An increase of bromodeoxyuridine (BrdU)-labeled cells is observed. The red fluorescent channel was inverted, making BrdU-labeled cells appear dark. Scale bar, $50 \mu \mathrm{m}$. Right: Quantification of labeled cells in the dentate gyrus after repeated ECSs. The y axis is cell counts per subgranular zone of the dentate gyrus. The number of proliferating cells increases with the number of ECSs administered, and five and 10 ECS sessions produced significant increases in the number of BrdU-labeled cells $\left(* * * p<.0001,{ }^{*} p<.005\right.$, compared with sham treatment; Bonferroni-Dunn correction. Error bars, SEM).

animal model of ECT. Maximal proliferation was observed at 3 to 5 days after the administration of a single ECS, in which the rate of proliferation had increased about threefold compared with control. The rate of cell proliferation correlated with the number of administered ECS. Newly generated neurons survived for at least 3 months. There was no evidence of increased apoptosis after single or multiple ECS.

Several groups have extended the original observation by Altman and Das (1965) that there is an ongoing neurogenesis in the adult hippocampus under physiologic conditions. Neurogenesis has been observed in a variety of species, including humans (Eriksson et al 1998; Gould et al 1998; Kempermann et al 1997b, 1998). In rodents, hundreds of new neurons are formed every day in the dentate gyrus (Gage et al 1998), and this number varies in response to different stimuli, including learning (Gould et al 1999), running (van Praag et al 1999), and changes in the environment (Kempermann et al 1997b). Recent evidence indicates that the newly formed neurons integrate functionally and show the same anatomical characteristics in the adult brain as do the older neurons (Markakis and Gage 1999).

Motor activity has been reported to be sufficient for inducing neurogenesis (van Praag et al 1999). This raises the possibility that the ictal motor activity is responsible for the effect observed here. We have previously shown, however, that seizure activity without motor convulsions can induce neurogenesis (Bengzon et al 1997). Furthermore, when ECS was given to animals under halothane anesthesia, thereby greatly reducing motor activity, a similar increase in BrdU-labeled cells was observed (Treschow et al, unpublished observations). These findings indicate that cerebral seizure activity per se is sufficient to induce neurogenesis. Other reports suggest that, under baseline conditions, newly generated neurons do not survive for very long in the adult brain and point to increased survival rather than increased proliferation as the key regulatory factor of adult neurogenesis (Gould et al 1999; Kempermann et al 1997b). In our study, we observed no pycnotic BrdU-labeled nuclei at 48 hours, 1 month, or three months after ECS. We did not find a decline in the number of BrdU-labeled cells after 3 months after ECS compared with 2 months. Furthermore, increased apoptosis was not observed after either a single or 10 sessions of ECS. Nonetheless, at present, we cannot exclude the possibility that some of the neurons generated by ECS die during the 1st month after treatment.

A decrease in hippocampal volume has been reported in humans after recurrent episodes of major depression (Shah et al 1998; Sheline et al 1996, 1999) and in posttraumatic stress disorder (Bremner et al 1995). Further, dysregulation of the glucocorticoid system and increased glucocorticoid levels have been observed in depressive disorders (Young et al 1991). In animals, exposure to increased levels of glucocorticoids causes atrophy and damage in hippocampal subfields (McEwen 1999). Glucocorticoid exposure also suppresses the rate of neuronal proliferation in the dentate gyrus of rats and decreases the length and arborization of apical dendrites of CA3 neurons (Magariños et al 1999), thereby augmenting a degenerative process. Thus, a dynamic balance between the formation of new neurons and neuronal degeneration seems to exist in the adult hippocampal formation.

There is no conclusive evidence linking hippocampal dysfunction and depressive disorders; however, comparing our experimental data with the clinical observation of a reduced hippocampal volume in severe depression and the striking therapeutic effect of ECT, we hypothesize that a stress-induced shift of this balance toward degeneration and decreased neuronal proliferation may be decisive in the neurobiological chain of events leading to the clinical syndrome of major depression. ECT may reverse this imbalance through a stimulation of neuronal proliferation. 
The amnestic symptoms accompanying ECT do not argue against this suggested hypothesis because they are transient and reversible (Squire 1986b). Furthermore, the memory disturbance of the untreated, depressed patient is a diminished acquisition of new information, whereas ECT causes a transient disruption of the retention of new information with unchanged or even increased acquisition. Once the series of ECT is completed, the capacity for new learning and retention, which depends on an intact hippocampal function, recovers in about 72 days (Weeks et al 1980). The long-term effect of ECT in depression is, therefore, not compromised by the reversible treatmentinduced memory disruption.

The reports of decreased hippocampal volume and possible concomitant atrophy in major depression, combined with the demonstration of increased neurogenesis in a treatment model for this disorder, add a new dimension to the conceptualization concerning the working action of ECT. The antidepressant effect of ECT may depend, at least partly, on the stimulation of cellular and synaptic plasticity in the hippocampal formation. Consistent with this idea, we previously discovered a sustained increase in the neuronal cell adhesion molecule (N-CAM), which is involved in synaptogenesis, following a series of ECS in rats treated under comparable experimental conditions (Jorgensen and Bolwig 1979). The results reported here suggest that new neural circuits are not only formed between preexisting neurons, but may also involve a large number of newborn neurons.

Further studies should clarify the possible role of the hippocampal function in the development of depressive disorders, especially with regard to the cognitive deficits found in depression.

This study was supported by the Danish and Swedish MRCs, the Theodore and Vada Stanley Foundation, the Ivan Nielsen Foundation, the Segerfalk Foundation, the Crafoord Foundation, the Zoega Foundation, the Wiberg Foundation, and the Kock Foundation. The authors thank Birgit H. Hansen for technical assistance.

\section{References}

Altman J, Das GD (1965): Autoradiographic and histological evidence of postnatal hippocampal neurogenesis in rats. J Comp Neurol 124:319-335.

American Psychiatric Association TFoET (1990): The Practice of Electroconvulsive Therapy: Recommendations for Treatment, Training, and Privileging. Washington, DC: American Psychiatric Association.

Bengzon J, Kokaia Z, Elmer E, et al (1997): Apoptosis and proliferation of dentate gyrus neurons after single and intermittent limbic seizures. Proc Natl Acad Sci U S A 94:1043210437.

Bremner JD, Randall P, Scott TM, et al (1995): MRI-based measurement of hippocampal volume in patients with combat-related posttraumatic stress disorder. Am J Psychiatry 152:973-981.

Devanand DP, Dwork AJ, Hutchinson ER, et al (1994): Does ECT alter brain structure? Am J Psychiatry 151:957-970.

Eriksson PS, Perfilieva E, Bjork-Eriksson T, et al (1998): Neurogenesis in the adult human hippocampus. Nat Med 4:1313-1317.

Gage FH, Kempermann G, Palmer TD, et al (1998): Multipotent progenitor cells in the adult dentate gyrus. $J$ Neurobiol 36:249-266.

Goodwin FK, Jamison KR (1990): Manic-Depressive Illness. New York: Oxford University Press.

Gould E, Beylin A, Tanapat P, et al (1999): Learning enhances adult neurogenesis in the hippocampal formation. Nat $\mathrm{Neu}$ rosci 2:260-265.

Gould E, Tanapat P, McEwen BS, et al (1998): Proliferation of granule cell precursors in the dentate gyrus of adult monkeys is diminished by stress. Proc Natl Acad Sci U S A 95:3168-3171.

Janicak PG, Davis JM, Gibbons RD, et al (1985): Efficacy of ECT: A meta-analysis. Am J Psychiatry 142:297-302.

Jorgensen OS, Bolwig TG (1979): Synaptic proteins after electroconvulsive stimulation. Science 205:705-707.

Kempermann G, Kuhn HG, Gage FH (1997a): Genetic influence on neurogenesis in the dentate gyrus of adult mice. Proc Natl Acad Sci U S A 94:10409-10414.

Kempermann G, Kuhn HG, Gage FH (1997b): More hippocampal neurons in adult mice living in an enriched environment. Nature 386:493-495.

Kempermann G, Kuhn HG, Gage FH (1998): Experienceinduced neurogenesis in the senescent dentate gyrus. $\mathrm{J} \mathrm{Neu}$ rosci 18:3206-3212.

Liu J, Solway K, Messing RO, Sharp FR (1998): Increased neurogenesis in the dentate gyrus after transient global ischemia in gerbils. $J$ Neurosci 18:7768-7778.

Magariños AM, Deslandes A, McEwen BS (1999): Effects of antidepressants and benzodiazepine treatments on the dendritic structure of CA3 pyramidal neurons after chronic stress. Eur J Pharmacol 371:113-122.

Markakis EA, Gage FH (1999): Adult-generated neurons in the dentate gyrus send axonal projections to field CA3 and are surrounded by synaptic vesicles. J Comp Neurol 406:449-460.

Mathé AA (1999): Neuropeptides and electroconvulsive treatment. J Electroconvuls Ther 15:60-75.

McEwen BS (1999): Stress and hippocampal plasticity. Annu Rev Neurosci 22:105-122.

Mongeau R, Blier P, de Montigny C (1997): The serotonergic and noradrenergic systems of the hippocampus: Their interactions and the effects of antidepressant treatments. Brain Res Rev 23:145-195.

Nibuya M, Morinobu S, Duman RS (1995): Regulation of BDNF and trkB mRNA in rat brain by chronic electroconvulsive seizure and antidepressant drug treatments. J Neurosci 15: 7539-7547.

Parent JM, Janumpalli S, McNamara JO, Lowenstein DH (1998): Increased dentate granule cell neurogenesis following amygdala kindling in the adult rat. Neurosci Lett 247:9-12.

Paxinos G, Watson C (1986): The Rat Brain in Stereotactic Coordinates. Sydney: Academic Press Australia. 
Sackeim HA, Luber B, Katzman GP, et al (1996): The effects of electroconvulsive therapy on quantitative electroencephalograms. Relationship to clinical outcome. Arch Gen Psychiatry 53:814-824.

Shah PJ, Ebmeier KP, Glabus MF, Goodwin GM (1998): Cortical grey matter reductions associated with treatmentresistant chronic unipolar depression. Controlled magnetic resonance imaging study. Br J Psychiatry 172:527-532.

Sheline YI, Sanghavi M, Mintun MA, Gado MH (1999): Depression duration but not age predicts hippocampal volume loss in medically healthy women with recurrent major depression. J Neurosci 19:5034-5043.

Sheline YI, Wang PW, Gado MH, et al (1996): Hippocampal atrophy in recurrent major depression. Proc Natl Acad Sci U S A 93:3908-3913.

Squire LR (1986a): Mechanisms of memory. Science 232:16121619.
Squire LR (1986b): Memory functions as affected by electroconvulsive therapy. Ann N Y Acad Sci 462:307-314.

Vaidya VA, Siuciak JA, Du F, Duman RS (1999): Hippocampal mossy fiber sprouting induced by chronic electroconvulsive seizures. Neuroscience 89:157-166.

van Praag H, Kempermann G, Gage FH (1999): Running increases cell proliferation and neurogenesis in the adult mouse dentate gyrus. Nat Neurosci 2:266-270.

Weeks D, Freeman CP, Kendell RE (1980): ECT: III: Enduring cognitive deficits? Br J Psychiatry 137:26-37.

Wolf HK, Buslei R, Schmidt-Kastner R, et al (1996): NeuN: A useful neuronal marker for diagnostic histopathology. J Histochem Cytochem 44:1167-1171.

Young EA, Haskett RF, Murphy-Weinberg V, Watson SJ, Akil H (1991): Loss of glucocorticoid fast feedback in depression. Arch Gen Psychiatry 48:693-699. 